\title{
Article
}

\section{Pathological human astroglia in Alzheimer's disease: opening new horizons with stem cell technology}

Mohamet, Lisa, Jones, Vicky Claire, Dayanithi, Govindan and Verkhratsky, Alexei

Available at http://clok.uclan.ac.uk/23029/

Mohamet, Lisa, Jones, Vicky Claire ORCID: 0000-0001-8401-5223, Dayanithi, Govindan and Verkhratsky, Alexei (2018) Pathological human astroglia in Alzheimer's disease: opening new horizons with stem cell technology. Future Neurology, 13 (2). pp. 87-99. ISSN 1479-6708

It is advisable to refer to the publisher's version if you intend to cite from the work. http://dx.doi.org/10.2217/fnl-2017-0029

For more information about UCLan's research in this area go to http://www.uclan.ac.uk/researchgroups/ and search for <name of research Group>.

For information about Research generally at UCLan please go to http://www.uclan.ac.uk/research/

All outputs in CLoK are protected by Intellectual Property Rights law, including Copyright law. Copyright, IPR and Moral Rights for the works on this site are retained by the individual authors and/or other copyright owners. Terms and conditions for use of this material are defined in the policies page.

\section{CLoK}

Central Lancashire online Knowledge www.clok.uclan.ac.uk

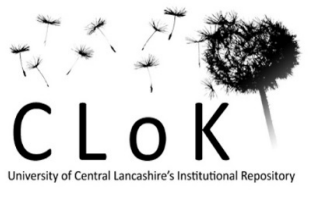




\title{
Pathological human astroglia in Alzheimer's disease: opening new horizons with stem cell technology
}

\author{
Lisa Mohamet $^{1}$, Vicky C Jones ${ }^{2}$, Govindan Dayanithi ${ }^{3,4}$ \& Alexei Verkhratsky ${ }^{5,6}$, \\ ${ }^{1}$ StrataStem Ltd, 11 Riverview, The Embankment Business Park, Cheshire, SK4 3GN, \\ ${ }^{2}$ School of Pharmacy and Biomedical Sciences, The University of Central Lancashire, \\ Preston PR1 2HE, UK; ${ }^{3}$ Department of Molecular Neurophysiology, Institute of \\ Experimental Medicine, Czech Academy of Sciences, 14220 Prague, Czech Republic, \\ ${ }^{4}$ Ecole Pratique des Hautes Etudes, Sorbonne, Les Patios Saint-Jaques, 75014 Paris, \\ France, ${ }^{5}$ Faculty of Biology, Medicine and Health, The University of Manchester, \\ Manchester, UK, ${ }^{6} I K E R B A S Q U E$, Basque Foundation for Science, 48011, Bilbao, \\ Spain and Department of Neurosciences, University of the Basque Country \\ UPV/EHU, 48940, Leioa, Spain.
}

Running head: Human astrocytes in AD

*Send correspondence to:

Dr. Lisa Mohamet

StrataStem Ltd,

11 Riverview,

The Embankment Business Park,

Cheshire, SK4 3GN, UK

Email: lisamohamet@stratastem.com

OR

Prof. Alexei Verkhratsky

The University of Manchester, Oxford Road, Manchester, M13 9PT, UK.

Telephone +44 (0)161-2757324,

E-mail: Alexej.Verkhratsky@manchester.ac.uk

First draft submitted: 13-Oct-2017; Accepted for publication: 24-Jan-2018; Published online: TBC 


\begin{abstract}
Pathological remodelling, degeneration and reactivity of astrocytes are fundamental astrogliopathies contributing to all neurological diseases. In neurodegenerative disorders (including Alzheimer's disease, AD) astroglia undergo complex changes that range from atrophy with loss of function to accumulation of reactive cells around disease-specific lesions (senile plaques in the case of AD). The cellular pathology of astroglia in the context of human AD remains enigmatic; mainly because of severe limitations of animal models, which, although reproducing some pathological features of the disease, do not mimic its progression in full. Human induced pluripotent stem cells technology creates a novel and potentially revolutionising platform for studying fundamental mechanisms of the disease and for screening to identify new therapeutic compounds.
\end{abstract}

Key words: Alzheimer's disease; astrocyte; astroglial atrophy; induced pluripotent stem cells; glutamate transporter EAAT1, GFAP, protein S100B, astrogliopathology 


\section{Astroglia: Homeostatic and defensive cells of the CNS}

Astrocytes ( $\alpha \sigma \tau \rho \circ v \kappa v \tau o \varsigma$ or star-like cells [1]) are a highly heterogeneous class of neural cells residing in the brain and spinal cord. Astrocytes are the major homeostatic cells of the CNS, executing their diverse functions at molecular, cellular, tissue and organ levels; furthermore astrocytes contribute to several systemic functions such as regulation of $\mathrm{Na}^{+}$balance, energy homoeostasis and food intake [2-5]. Multiple homoeostatic pathways expressed in astroglial cells represented, for example, by membrane transporters for neurotransmitters [6], a complex system of secretion of neuromodultors, neurotransmitter precursors and hormones [7] or aerobic glycolysis producing energy substrates [8] maintain CNS functional activity and provide essential neuroprotection.

Astrocytes are integrated into functional syncytia by gap junctions formed by connexions $\mathrm{Cx} 43$ and $\mathrm{Cx} 36$ and these gap junctional channels, or connexons, are permeable to hydrophilic molecules with molecular weight $<1 \mathrm{kDa}$ thus allowing intercellular transfer of ions, second messengers, nucleotides, glutathione, prostaglandins, metabolites, vitamins and RNA [9]. Astroglial syncytial networks are segregated within defined anatomical structures; for example, astroglial syncytia are limited to individual barrels in the somatosensory cortex [10]. Astrocytes establish heterocellular contacts with oligodendrocytes, which are likely important for ion homoeostasis in the white matter [11]. Thus astrocytes represent the "reticular" portion of the CNS and can provide for functional integration of neuronal ensembles and synaptic fields. In the grey matter astrocytes occupy non-overlapping territorial domains, therefore dividing the nervous tissue into relatively independent glio-neurovascular units that bridge neurons with vasculature and regulate functional hyperemia [12]. Astroglial endfeet plaster capillaries and form perivascular space that is a key element of the CNS-wide glymphatic system that removes excreted proteins and toxic molecules from the interstitium by the virtue of convectional fluid flow $[13,14]$.

Within their territorial domains astroglial membranes cover neuronal elements. In particular perisynaptic astroglial processes enwrap the majority of excitatory synapses in the brain, forming the 'astroglial cradle' critical for synaptogenesis, synaptic maturation, synaptic maintenance and extinction [15]. Astrocytes control neurotransmitter dynamics in the CNS through neurotransmitter removal and subsequent catabolism. Astrocytes, for example, specifically express adenosine kinase, which is the key enzyme for adenosine degradation [16] and monoaninoxidase-B, which the major enzyme of catabolism of catecholamines [17]. Astroglial cells are a central element of glutamate/GABA-glutamine shuttle that supplies neurones with glutamine, an indispensable precursor of glutamate and GABA. Operation of this shuttle is supported by astrocyte plasmalemmal expression of transporters for glutamate, GABA and glutamine, and by the astroglia-specific enzyme glutamine synthetase [18].

The defensive capabilities of astroglia are defined by their homeostatic pathways and by their evolutionary conserved defence response, known as reactive astrogliosis. Astrocytes protect neurones through glutamate clearance and $\mathrm{K}^{+}$buffering (which curtail excitotoxicity), through supplying neurones with energy substrates or through provision of reactive oxygen species scavengers [19]. Insults to the CNS (of various 
aetiology and acuteness) trigger a complex remodelling of astrocytes, which results in multiple "reactive" phenotypes that can be neuroprotective as well as neurotoxic [2023]. Furthermore, astroglial reactivity is, most likely, context and disease-specific, which further increases the number of possible reactive phenotypes [24]. Although, at its very core, reactive astrogliosis is a defensive response, it may acquire (for example during chronic or excessive activation) mal-adaptive dimensions [24, 25].

\section{Specific properties of human astroglia}

The evolution of astroglia arguably reaches its summit in the brain of humans and higher primates [5]. The protoplasmic and fibrous astrocytes of the human brain are substantially (10 - 20 times) larger and protoplasmic astrocytes are substantially more complex than the astrocytes of rodents. As a result, a human protoplasmic astroglial cell covers $\sim 20$ times more synapses than the same cell in the rodent brain [26, 27]. In addition, human brains contain several unique types of astrocytes, which are absent in non-primate CNS and which extend one or more exceptionally long processes, in the order of millimetres. A rather abundant type of human astroglia (which may account for $\sim 10 \%$ of all astroglial cells in the human brain) is represented by interlaminar astrocytes, discovered at the end of $19^{\text {th }}$ century $[28,29]$. These cells have relatively small bodies $(\sim 10 \mu \mathrm{m})$ localised to cortical layer I, several short and one or two millimetre-long processes, which penetrate through layers II to IV and terminate with characteristic bouton-like structures known as terminal masses or end bulbs $[26,29]$. A second type of astroglia, found only in humans and higher primates are polarised astrocytes. Somata of these astrocytes are positioned in the deep cortical layers close to the white matter; these cells send two exceptionally long ( 1 to $5 \mathrm{~mm}$ in length) processes that penetrate into superficial cortical layers [26]. Finally, human brains have a peculiar astrocyte subset, generally similar to protoplasmic astroglia with several very long (up to $1 \mathrm{~mm}$ ) unbranched processes with evenly spaced varicosities that extend in all directions throughout the cortex, many of which are in contact with blood vessels [26, 30]. These astrocytes never appear in the brains of neonates, and the density of these astrocytes varies substantially between individuals [30].

Beyond morphological differences, further heterogeneity is revealed by the relative protein expression profiles human astroglia. Whilst all of the human astroglial subtypes outlined above are positive for canonical astrocyte marker, glial fibrillary acidic protein (GFAP), their expression of other astrocyte-associated proteins such as the calcium binding protein, S100B, excitatory amino acid transporters 1 and 2 (EAAT1/2) and glutamine synthetase vary markedly [26, 30]. Indeed, interlaminar astrocytes, as well as varicose projection astrocytes for example, are unique in their reactivity to antibodies against the extracellular matrix receptor, CD44 [31, Sosunov, 2014 \#29]

Is there a possible link between specific morphology of human astrocytes and cognitive superiority of the human brain? Recent experiments have demonstrated that grafting of foetal human glial progenitor cells (hGPCs) into the brains of young immunosuppressed mice resulted in a substantial change in neural networking. Astrocytes derived from these progenitors-derived astrocytes readily expanded and populated large portions of the mouse brain, subsequently replacing host astroglia yet retaining their human-specific morphology and complexity [32, 33, Goldman, 2015 
\#95]. This "humanised" rodent brain demonstrated higher cognitive abilities as measured by improved memory, novel object recognition and auditory fear conditioning compared to wild type controls [32], further highlighting the enhanced capabilities of human astroglia comparative to rodent. Nonetheless, the underlying mechanisms for cognitive improvement remain to be discovered: these may indeed be associated with higher integrative capabilities of human astrocytes or reflect higher homeostatic capacity of the latter.

\section{Principles of astrogliopathology}

Diseases of the CNS can be generally defined as a homeostatic failure of nerve tissue, and hence they are directly associated with the functional performance of homeostatic neuroglia. Astrocyte pathology contributes to all types of neurological disorders. This contribution is complex and disease-specific; moreover, different astrogliopathic changes may develop simultaneously or sequentially during pathological evolution of neurological disorders. Conceptually, pathological changes of astroglia can be classified into astrodegeneration and asthenia with loss of function, pathological remodelling of astroglia and reactive astroglial response [19, 24, 25, 34]. Astrodegeneration and loss of function dominate, for example in neuropsychiatric disorders [35], astroglial pathological remodelling is predominant in Alexander disease or in some forms of epilepsy [36, 37], whereas astroglial reactivity assumes the leading role in neurotrauma and stroke $[21,38]$.

\section{Astrocytes in Alzheimer's disease}

Neurodegenerative disorders reflect chronic extinction of neural tissue, which results in specific lesions and symptoms, such as impaired locomotive control in Parkinson's disease, or loss of motor neurones in amyotrophic lateral sclerosis and in more generalised cognitive downfall that ultimately terminates in dementia. The role for neuroglia in these diseases is yet to be fully understood. Notwithstanding, glial contribution to neuropathophysiology is receiving growing attention and the notion that the progression of neurodegenerative processes is defined, to a large extent, by the capability of glia to resist pathology and to compensate for functional failures gains wider acceptance [34, 39-45].

In Alzheimer's disease (AD), astroglia undergo complex and regionally-specific pathological changes. A decrease in astroglial profiles (indicative of atrophic changes) at the early stages of disease progression have been observed in studies on transgenic animals harbouring AD-related mutant human genes [46-50]. This coincided with suppressed astrogliosis in entorhinal and prefrontal cortices that may underlie high vulnerability of these regions to $\mathrm{AD}$ pathology $[45,48,50]$. At the later stages of disease, emergence of senile plaques elicited astroglial reactivity, with hypertrophic cells accumulating around the plaques [49, 51]. In the human brain, astrogliosis is prominent at the early stages of the disease, but decreases in advanced stages of $\mathrm{AD}$, when overall glial paralysis contributes to severe brain atrophy [45, 52]. In summary, various aspects of astrogliopathic changes contribute to the progression of $\mathrm{AD}$, and conceivably astrocytes define both early cognitive deficits due to deficient synaptic support while astroglial reactivity defines resilience of nervous tissue to the pathology. 


\section{Limitations of current models: The need for humanising astroglial research}

Progress in understanding AD has been hindered by the lack of suitable disease models $[53,54]$, reflected in an inability to translate results from animal studies to successful human therapies. The human pathology is dominated by the late-onset or sporadic form of $\mathrm{AD}$ (SAD); which disease variant does not display significant Mendelian genetic bias. AT the same time absolute majority of cell- and animal-based models of $\mathrm{AD}$ employ mutant genes isolated from the clinically rare, dominantly inherited familial AD (FAD) characterised by the early onset. Neuronal loss and cognitive deficits, which are the hallmarks of AD in humans, are rather limited in animal models [55]. Very little is known about pathological astroglial phenotypes in the context of human $\mathrm{AD}$, again mainly because of limitations of animal models; in particular remarkable differences between rodent and human astrocytes, hinder in depth characterisation of translational pathophysiology of human astroglia.

Another significant barrier to studying human astrocytes is the difficulty of accessing and culturing sufficient astrocytes from the mature human brain. The current state-ofthe-art involves isolation of a proliferative progenitor cell that can be subsequently matured in vitro. However, these cells can quickly become quiescent in long-term cultures and when cultured in the presence of serum, show a morphological phenotype markedly different to those in vivo. A more recently developed technique known as 'immunopanning' provides improved isolation and purification of mature human astrocytes [56]. The 'immunopanned' cells were used to assess gene expression signatures in human and mouse astrocytes showing that only a third of the genes most enriched in human astrocytes were similarly expressed in mouse [56]. Of further significance, is that the authors also demonstrated functional hominid distinctions to rodents highlighted by differences in astroglial responses to exogenous glutamate. Nonetheless, these types of invasive methods for human adult astrocyte sample collection remain challenging. Traditional cell-based approaches utilise assays based on primary cells and/or transformed cell lines, which fail to offer a physiologically relevant in vitro model that captures the specific genomic information of the patient or the biology for complex diseases of ageing with environmental and genetic risk factors, such as AD. Together, these limitations underscore a pressing need for new technologies that can replicate human (patho)physiology to provide means for improved disease modelling and a better understanding of human astrocyte development.

\section{A New Platform for Human Astrocyte Research and Drug Discovery}

Human induced pluripotent stem cells (iPSCs) are generated via reprogramming somatic cells with a number of key transcription factors that are crucial to maintaining undifferentiated embryonic stem cells (also termed pluripotent stem cells) [57-59]. This creates an ideal physiologically-relevant model because cells (i) can be derived from any individual; (ii) are genetically identical to the donor; (iii) can self-renew indefinitely and (iv) have the capacity to form any cell type of the body. Therefore providing a platform that offers significant advantages over existing models, by delivering the only source of clinically relevant, healthy and diseased human cell types amenable to various regenerative medicine applications (Fig. 1). 
The continued progress of iPSC technology over the last decade has provided improved biologically relevant models, yet there are still considerable hurdles to overcome. Of particular relevance is the need for robust and cost-effective methods for the differentiation of iPSCs into mature major cell types of the CNS (such as neurones or astrocytes). Recent studies have shown that neurones differentiated from SAD- and FAD-iPSCs resemble pathologically affected cells in vivo and express key disease hallmarks [60-62], However, there is still a significant demand for tools that provide robust, homogeneous astroglial populations to help reveal astroglial contribution to neurodegenerative diseases. Despite a plethora of publications indicating the generation and utility of iPSC-derived neurones for disease modelling to date, in comparison only a limited number of studies have described consistent generation of enriched astrocytes from healthy donors [63-68]. Generation of functional astroglia from healthy iPSCs has previously been reported to be timeconsuming (60 - 90 days post differentiation) with further limitations in purity (cultures show 20 - 30\% contamination with unwanted cell types) [63-65, 69], which denotes a significant challenge in delineating autonomous contribution of astroglia in disease pathogenesis. Current protocols exploit the gliogenic switch observed during embryonic development in vivo, whereby human iPSC-derived astroglia can be derived from either patterned neural stem cells (NSCs) or from committed glial lineage precursors that represent an intermediate population of progenitors [70, 71]. This directed differentiation relies on the activation of a number of key signal transduction pathways that contribute to astroglial differentiation, proliferation and maturation at specific time points. We have recently demonstrated [72] highly efficient generation of enriched populations of mature human cortical astroglia (with less than 5\% of neurone contamination) from independent donor iPSC lines, within 30 days of induction from cortical NSCs. This technique adapted a chemically-defined differentiation protocol [63] in which NSCs were exposed to ciliary neurotrophic factor (CNTF), which is known to induce astrocyte formation in cerebral cortical precursors by activation of downstream JAK/STAT signalling that transcriptionally activate astrocyte specific loci, such as GFAP and S100B[73]; and bone morphogenetic protein 2 (BMP2), which has also been shown to drive astrocyte differentiation from NSCs [74, 75]. In order to augment astrocyte formation, the media was supplemented with exogenous mitogens, epidermal growth factor (EGF) and insulin, which have been shown to increase glial commitment, primary astrocyte proliferation and sub-culturing in vitro $[76,77]$. A principal schematic diagram of astroglial differentiation from human iPSCs is presented in Fig. 2 A - C.

Astrocytes derived from human iPSCs exhibit typical morphology associated with primary astroglial cells in vitro and express several archetypal markers (Fig. 2C). Moreover, healthy iPSC-derived astroglia show significant morphological heterogeneity, arguably reflecting well-characterised astroglial heterogeneity in vivo $[26,30,78,79]$. The iPSC-derived astrocyte populations showed robust expression of GFAP, a classical marker of astrogliosis that is always up-regulated in vitro [80]. Similarly, iPSC-derived astroglia have been found to express other canonical markers, such as CD44 antigen, S100B or aldehyde dehydrogenase1 family member L1, ALDH1L1 and several astroglia-specific molecules critical for astroglial homeostatic function, such as, glutamate transporter EAAT1 and EAAT2 and glutamine synthetase [71, 72]. Additional functional studies have shown that iPSC-derived astroglia derived from human iPSCs are capable of generating spontaneous $\mathrm{Ca}^{2+}$ signals $[71,81]$, have phagocytic activity and mount neuroimmunological response 
[81], although detailed physiological characterisation of these cells is yet to be produced. Of further interest is the characterisation of astroglial regional subtypes throughout the CNS. While this is limited by incomplete knowledge of robust markers to delineate such cells, there is some evidence to suggest that maturation of neurones or glia from regionally specified iPSC-derived NPCs maintain their regional identity [82]. Together these data validate the use of iPSC models for interrogation of human astroglial function and development, which to date, is still largely unknown.

\section{Patient iPSC-derived astroglia exhibit AD-related pathological signatures}

Astroglia contribute to non-cell autonomous mechanisms in various neurodegenerative disorders that were classically considered exclusively neuronal diseases [34, 83]. Although studies of cell-autonomous pathobiology of human astrocytes derived from patient stem cells are in the nascent state, several lines of evidence show that these astroglia retain some pathological disease signatures, including in $\mathrm{AD}$ (Table 1).

Human astroglia reprogrammed from dermal fibroblasts of a 53 year old male donor with type III early onset FAD (bearing an M146L mutation in the presenellin-1 gene, PSEN1) and from an 87 year old female clinically affected with late-onset SAD (homozygous for the 4 allele of apolipoprotein E, $\mathrm{ApoE}^{+/+}$; the single-largest genetic factor determining SAD risk) exhibited morpho-pathological phenotypes when compared with iPSC-derived astroglia reprogrammed from a healthy control [72]. This pathological signature comprised of (i) mislocalisation and abnormal expression of mature astrocyte markers, (ii) compromised astrocyte heterogeneity and (iii) astroglial atrophy. Astrocytic dysgenesis, manifested as an almost complete loss of processes and overall reduction in cell size was significant (Fig. 3); this finding strongly correlates with observations of morphological astroglial atrophy in early stage AD pathology in mouse models [45, 49]. In contrast, astrocytes derived from iPSCs isolated from patients with fronto-temporal dementia show hypertrophic morphology [69] further indicating disease-specific glial metamorphoses.

Distribution of astroglial markers was similarly aberrant in cells derived from AD patients. For example, S100B in AD iPSC-derived astroglia was almost entirely confined to large nuclear inclusions, in contrast to healthy iPSC-derived astroglia, which show typical cytoplasmic localisation. This was accompanied by significant decrease in total $\mathrm{S} 100 \mathrm{~B}$ expression in $\mathrm{AD}$-astroglia when compared to control astroglia. Since S100B is known to interact with various cytoskeletal components, this phenomenon may represent a novel and early mechanism underlying SAD- and FADinduced astrocytic atrophy. Mislocalisation was also observed for glutamine synthetase in both FAD and SAD- astroglia, while the glutamate transporter EAAT1 was misplaced only in SAD iPSC-derived astroglia. Furthermore, the expression levels of both EAAT1 and glutamine synthetase were decreased only in FAD-derived astroglia [72]. A recent study using iPSC-derived astrocytes from donors with PSEN1 $\Delta 9$ mutation failed to show differences in expression of S100B, EAAT-1 and -2 transcripts compared to healthy donors [84]. However, these differences can be attributed to the fact that protein mislocalisation and discrete changes in protein levels might not be captured at the transcript level. Intriguingly, other iPSC-based studies have shown that astroglia derived from iPSCs from SAD and FAD backgrounds as well as from healthy individuals carrying $A P O E 4$, exhibit no significant deficit in 
their overall abilities to sequester extracellular glutamate compared to controls [66, 85]. Whether these findings are a result of the application of glutamate assay concentrations being orders of magnitude higher than predicted physiological levels [86], and hence masking nuanced alterations in uptake, or whether they imply that it is the processing rather than gross uptake of glutamate that is altered in $\mathrm{AD}$, remains to be established. Astrocytes derived from iPSCs obtained from AD patients with PSEN1 $\triangle E 9$ mutation demonstrated aberrant $\mathrm{Ca}^{2+}$ signaling and altered release of cytokines [84].

Accumulation of $\beta$-amyloid oligomers in intracellular organelles has been described in some, but not in all, iPSC-derived SAD- and FAD- astrocytes, concomitant with the induction of both ER and oxidative stress [66]. Specifically, those astrocytes derived from patients with the $A P P$-E693 $\triangle$ mutation demonstrated large inclusions of $\beta$-amyloid, which were shown to co-localise to the endoplasmic reticulum, early endosomes and lysosomes, while those from $A P P-\mathrm{V} 717 \mathrm{~L}$ patients showed no accumulation at all. Increased astroglial $\beta$-amyloid production was also observed in astrocytes derived from patients with PSEN1 $\triangle \mathrm{E} 9$ mutation [84]. These findings shed new insight into disease heterogeneity, both within and between SAD and FAD, and are supportive of our own findings of subtle variations at the cellular level between the two forms of the disease [72]. At the same time, production of $\beta$-amyloid as well as soluble amyloid precursor protein- $\alpha$ was detected in astrocytes derived from healthy human iPSCs, possibly hinting on the role of astroglia in amyloidogenesis [67].

Out of three human isoforms of apolipoprotein homozygous expression of the E4 alleles confers significantly increased risk for late-onset SAD [87]. In contrast, the E2 isoform has been reported to confer a protective mechanism against $\mathrm{AD}$; and the most common, E3 isoform appears pathologically neutral [88]. While it is known that ApoE contributes to neuronal health by transporting the cholesterol required for cellular repair, synaptic plasticity and dendritic spine integrity [89], the precise mechanisms by which the ApoE isoforms contribute to AD pathogenesis or protection are not fully understood. Astroglia generated from iPSCs derived from ApoE4 ${ }^{+/+}$ donors secrete active ApoE, but exhibited profound hypolipidation in astrocytes ApoE4 $^{+/+}$SAD-prone background compared to ApoE3 $3^{+/+}$controls (Zhao, 2017 \#90, consistent with findings in mice and humans [90,91]. Thus, human iPSC-derived astrocyte models provide an excellent platform to study relative contribution of its isoforms to $\mathrm{AD}$ progression.

Of further interest is that iPSC-derived ApoE4 ${ }^{+/+}$astrocytes show impaired abilities to support neuronal survival and synaptogenesis, compared to healthy iPSC-derived astrocytes; indicating a loss of neurotrophic and neuroprotective functions [85]. This functional deficit appears independent of neuronal contact or glutamate scavenging, hence implicating alterations in the astrocytic secretome. Indeed, we revealed significant alteration of the constitutive secretion of the pro-inflammatory mediators IL-8 (CXCL8) and MCP-1 (CCL2) in FAD- and SAD iPSC-derived astroglia when compared to healthy iPSC-derived astroglia [72]. These observations may further indicate a potential glial paralysis that has been postulated to be a fundamental factor in the evolution of $\mathrm{AD}$ [45]. In summary, astroglia derived from iPSCs are capable of mimicking in vitro a wide variety of deficits in homeostatic molecular cascades 
intimately associated with AD that are routinely observed in vivo and in patients [9295].

Harnessing iPSC technology will also provide a novel platform for accelerated drug discovery. Recent proof of concept studies have demonstrated iPSC-derived astrocytes as a promising cellular tool for high throughput compound screening [96] and for personalised treatment across a number of neurological disorders [66, 97, 98]. By testing whether drugs have differential effects in iPSC-derived astrocytes, we can begin to unravel how genetic variation and/or cellular phenotypes in AD can dictate responses to different drugs and help to elucidate novel targets.

\section{Conclusions}

Human astrocytes bear numerous idiosyncratic features that fundamentally distinguish them from astroglia in lesser mammals. Astroglia are a fundamental component to neurodegenerative diseases; hence studies of human astrocytes are of significant importance for further understanding of the mechanisms of neurodegeneration. The intent of this review was to broadly summarise recent progress in iPSC technology to develop novel cellular platforms for studying human astrocytes in the context of AD. Astroglia can be readily derived from somatic cells of patients suffering from AD; these cells express canonical astroglial markers and bear a number of pathological signatures. Further characterisation of the functional pathological phenotype of these cells is of ultimate importance for establishing a new human-based platform that will provide us with a better understanding of early changes in CNS homeostasis leading to $\mathrm{AD}$ that can be used in high throughput phenotypic screening assays to identify potential new therapeutic compounds that may provide a better clinical outcome for patients.

\section{Future perspectives}

"Humanisation" of neuropathological research is the main challenge which theoretical medicine is facing. A large majority of neurological diseases do not have an effective cure and with only symptomatic therapies available at best. There are classes of neurological disorders (most notably neuropsychiatric, neurodevelopmental and neurodgenerative diseases) which do not develop in animals (rare occurances of neurodegenrative phenotype in lemurs or in some canines remain exeptions), and hence require development of artificial animal models. These are commonly produced in model organisms such as mice, zebrafish and Drosophila due to their amenability for genetic manipulation. However, the brains of these animals are not even remotely close to the brain of humans, their lifespan is significantly shorter and thir social inetractions are much inferior and fundamentally distinct to humans. These limitations undrlie slow progress of neurological therapies.

Another salient revolution developing over last decade concerns a fundamental shift in the understanding of cellular pathophysiology of the brain. The classical paradigm that regards neurones as the cell-autonomous substrate of neuropathology has shifted towards neuroglial mechanisms, that, by virtue of homeostatic and defensive capabilities, seem to determine the resitivity of nervous tissue to pathological insults and chronic neuropathologies. Evolution of astroglia from lesser mammals to humans 
is remarkable and human astroglia are unique in their complexity compared to rodents. Poor translation from animal models to clinical outcomes has severely limited the development of effective therapeutics for neurological disorders to date, not least $\mathrm{AD}$ which remains incurable. The idiosyncratic astroglia of the human brain might be the key to better understanding of uniquely human neurological diseases; hence the development of such human-based models as described here to more accurately study such diseases is essential. Emerging evidence using iPSC astroglial models is beginning to uncover subtle variations in individual molecular and cellular phenotypes not only between but also within FAD and SAD classifications. Invariably these models pave the way towards the stratification of patient treatment regimes and personalised medicine.

However, as a note to the reader, it should be emphasised that cultured astrocytes represent a simplified model relative to that of astrocytes in the CNS, whereby additional interactions with other cell types and matrix components are likely to influence the astrocytic phenotype. Yet, highly purified human astrocyte cultures combined with recent advances in precision genome editing such as CRISPR/Cas9 represent a unique system to delineate the autonomic responses of astrocytes to defined stimuli/matrix/co-cultures in both healthy and AD-affected cells in an unparalleled manner.

\section{Financial \& competing interests disclosure}

The authors have no relevant affiliations or financial involvement with any organization or entity with a financial interest in or financial conflict with the subject matter or materials discussed in the manuscript. This includes employment, consultancies, honoraria, stock ownership or options, expert testimony, grants or patents received or pending, or royalties.

No writing assistance was utilized in the production of this manuscript.

\section{Executive summary}

- Astrocytes are a class of neuroglia primarily responsible for overall homeostasis of the nervous system.

- Astrocytes are now understood as key elements of virtually all neurological diseases and thus represent an understudied target for therapy development.

\section{Limitations of current models for studying astrocytes}

- Human astrocytes differ significantly from rodent counterparts, exhibiting complex heterogeneity and comprising multiple unique subtypes exclusive to the higher primates brain

- Studies performed using either wild-type or transgenic rodent astrocytes have inherently limited translational value for understanding the nuances of astrocytic roles in human disease.

- Opportunities for accessing and culturing mature human astrocytes are extremely limited. 


\section{Human induced pluripotent stem cells}

- Human induced pluripotent stem cells (iPSCs) are created by reprogramming adult somatic cells to a nascent stem-like state.

- iPSCs maintain the capacity to differentiate in vitro to all cell types of the body by exposing them to defined combinations of growth factors, cytokines, hormones and mitogens.

\section{A new platform for studying astrocyte biology}

- A number of studies have described directed differentiation of iPSCs to astrocytes in vitro.

- Recent technological advances have improved robustness, reduced timelines for astrocyte maturation and minimised contamination by unwanted cells such as neurones.

- Human iPSC-derived astrocytes exhibit typical astroglial morphology and heterogeneity, express mature astrocyte markers and perform a number of key astrocytic functions, validating them as models for the investigation of human astroglial function and development.

\section{iPSC-derived astrocytes as a model for studying pathophysiology}

- iPSCs are derived from somatic cells (e.g. skin fibroblasts) of patients with various pathologies and can subsequently be differentiated into astrocytes.

- Recent studies have demonstrated the derivation of astrocytes from AD patient iPSCs.

- AD patient iPSC-derived astrocytes display key features of disease phenotype, including morphological aberrance, cellular stress, loss of neurotrophic and neuroprotective functions, alterations in the secretome and impaired lipidation status.

- Notably, these cells retain the patient's own unaltered genome and express disease-associated genes at physiological levels, making them an excellent platform for investigating disease and testing new therapies.

\section{Conclusion}

- Patient iPSC-derived astrocytes represent an unrivalled model for the investigation of human astrocyte development, astrocyte pathophysiology in $\mathrm{AD}$ and for screening to identify new therapeutic compounds. 


\section{References}

Papers of special note have been highlighted as:

$\bullet \bullet$ of considerable interest

1. Lenhossék MV. Der feinere Bau des Nervensystems im Lichte neuester Forschung, 2nd edition. Fischer's Medicinische Buchhandlung H. Kornfield, Berlin. (1895).

2. $\quad$ Verkhratsky A, Nedergaard M. Physiology of astroglia. Physiol. Rev. 98 239389 (2018).

- Comprehensive account on astroglial physiology

3. Yang L, Qi Y, Yang Y. Astrocytes control food intake by inhibiting AGRP neuron activity via adenosine $\mathrm{A}_{1}$ receptors. Cell. Rep. 11(5), 798-807 (2015).

4. Gourine AV, Kasymov V, Marina $\mathrm{N}$ et al. Astrocytes control breathing through pH-dependent release of ATP. Science 329(5991), 571-575 (2010).

5. Verkhratsky A, Nedergaard M. The homeostatic astroglia emerges from evolutionary specialization of neural cells. Philos. Trans. R. Soc. Lond. B Biol. Sci. 371(1700), (2016).

6. Zhou Y, Danbolt NC. GABA and glutamate transporters in brain. Front. Endocrinol. (Lausanne) 4165 (2013).

7. Verkhratsky A, Matteoli M, Parpura V, Mothet JP, Zorec R. Astrocytes as secretory cells of the central nervous system: idiosyncrasies of vesicular secretion. EMBO J. 35(3), 239-257 (2016).

8. Pellerin L, Magistretti PJ. Sweet sixteen for ANLS. J. Cereb. Blood. Flow Metab. 32(7), 1152-1166 (2012).

9. Theis M, Giaume C. Connexin-based intercellular communication and astrocyte heterogeneity. Brain. Res. 1487 88-98 (2012).

10. Giaume C, Koulakoff A, Roux L, Holcman D, Rouach N. Astroglial networks: a step further in neuroglial and gliovascular interactions. Nat. Rev. Neurosci. 11(2), 87-99 (2010).

11. Orthmann-Murphy JL, Freidin M, Fischer E, Scherer SS, Abrams CK. Two distinct heterotypic channels mediate gap junction coupling between astrocyte and oligodendrocyte connexins. J. Neurosci. 27(51), 13949-13957 (2007).

12. Attwell D, Buchan AM, Charpak S, Lauritzen M, Macvicar BA, Newman EA. Glial and neuronal control of brain blood flow. Nature 468(7321), 232-243 (2010).

13. Iliff JJ, Lee $\mathrm{H}, \mathrm{Yu} \mathrm{M}$ et al. Brain-wide pathway for waste clearance captured by contrast-enhanced MRI. J. Clin. Invest. 123(3), 1299-1309 (2013).

14. Nedergaard M. Neuroscience. Garbage truck of the brain. Science 340(6140), 1529-1530 (2013).

15. Verkhratsky A, Nedergaard M. Astroglial cradle in the life of the synapse. Philos. Trans. R. Soc. Lond. B Biol. Sci. 369(1654), 20130595 (2014).

16. Boison D, Chen JF, Fredholm BB. Adenosine signaling and function in glial cells. Cell Death Differ. 17(7), 1071-1082 (2010).

17. Saura J, Kettler R, Da Prada M, Richards JG. Quantitative enzyme radioautography with ${ }^{3} \mathrm{H}-\mathrm{Ro}$ 41-1049 and ${ }^{3} \mathrm{H}-\mathrm{Ro}$ 19-6327 in vitro: localization and abundance of MAO-A and MAO-B in rat CNS, peripheral organs, and human brain. J. Neurosci. 12(5), 1977-1999 (1992). 
18. Hertz L. The glutamate-glutamine (GABA) cycle: importance of late postnatal development and potential reciprocal interactions between biosynthesis and degradation. Front. Endocrinol. (Lausanne) 459 (2013).

19. Verkhratsky A, Sofroniew MV, Messing A et al. Neurological diseases as primary gliopathies: a reassessment of neurocentrism. ASN Neuro. 4(3), (2012).

20. Liddelow SA, Guttenplan KA, Clarke LE et al. Neurotoxic reactive astrocytes are induced by activated microglia. Nature 541(7638), 481-487 (2017).

21. Burda JE, Sofroniew MV. Reactive gliosis and the multicellular response to CNS damage and disease. Neuron 81(2), 229-248 (2014).

22. Pekny M, Pekna M. Astrocyte reactivity and reactive astrogliosis: costs and benefits. Physiol. Rev. 94(4), 1077-1098 (2014).

23. Sofroniew MV. Astrocyte barriers to neurotoxic inflammation. Nat. Rev. Neurosci. 16(5), 249-263 (2015).

24. Pekny M, Pekna M, Messing A et al. Astrocytes: a central element in neurological diseases. Acta Neuropathol. 131(3), 323-345 (2016).

- othis article sets up principles of astrogliopathology and classifies astrogliopathies

25. Verkhratsky A, Rodriguez JJ, Parpura V. Astroglia in neurological diseases. Future Neurol. 8(2), 149-158 (2013).

26. Oberheim NA, Takano T, Han X et al. Uniquely hominid features of adult human astrocytes. J. Neurosci. 29(10), 3276-3287 (2009).

- this paper provides a detailed comparison of the features of human astroglia compared to those of rodents

27. Oberheim NA, Goldman SA, Nedergaard M. Heterogeneity of astrocytic form and function. Methods Mol. Biol. 814 23-45 (2012).

$\bullet \bullet$ this paper provides a detailed comparison of the features of human astroglia compared to those of rodents

28. Andriezen WL. The neuroglia elements of the brain. Br. Med. J 2 227-230 (1893).

29. Colombo JA. The interlaminar glia: from serendipity to hypothesis. Brain Struct. Funct. doi:10.1007/s00429-016-1332-8 (2016).

30. Sosunov AA, Wu X, Tsankova NM, Guilfoyle E, Mckhann GM, 2nd, Goldman JE. Phenotypic heterogeneity and plasticity of isocortical and hippocampal astrocytes in the human brain. J. Neurosci. 34(6), 2285-2298 (2014).

31. Akiyama H, Tooyama I, Kawamata T, Ikeda K, Mcgeer PL. Morphological diversities of CD44 positive astrocytes in the cerebral cortex of normal subjects and patients with Alzheimer's disease. Brain Res. 632(1-2), 249-259 (1993).

32. Han X, Chen M, Wang F et al. Forebrain engraftment by human glial progenitor cells enhances synaptic plasticity and learning in adult mice. Cell Stem Cell 12(3), 342-353 (2013).

- the first demonstration of the ability of human astroglia to integrate into existing rodent brain circuits, and to enhance rodent cognition.

33. Windrem MS, Schanz SJ, Morrow C et al. A competitive advantage by neonatally engrafted human glial progenitors yields mice whose brains are chimeric for human glia. J. Neurosci. 34(48), 16153-16161 (2014).

34. Ferrer I. Diversity of astroglial responses across human neurodegenerative disorders and brain aging. Brain Pathol. 27(5), 645-674 (2017). 
35. Verkhratsky A, Rodriguez JJ, Steardo L. Astrogliopathology: a central element of neuropsychiatric diseases? Neuroscientist 20(6), 576-588 (2014).

36. Messing A, Brenner M, Feany MB, Nedergaard M, Goldman JE. Alexander disease. J. Neurosci. 32(15), 5017-5023 (2012).

37. Bedner P, Dupper A, Huttmann K et al. Astrocyte uncoupling as a cause of human temporal lobe epilepsy. Brain 138(Pt 5), 1208-1222 (2015).

38. Burda JE, Bernstein AM, Sofroniew MV. Astrocyte roles in traumatic brain injury. Exp. Neurol. 275 Pt 3 305-315 (2016).

39. Verkhratsky A, Zorec R, Rodriguez JJ, Parpura V. Astroglia dynamics in ageing and Alzheimer's disease. Curr. Opin. Pharmacol. 26 74-79 (2016).

40. Rodriguez JJ, Verkhratsky A. Neuroglial roots of neurodegenerative diseases? Mol. Neurobiol. 43(2), 87-96 (2011).

41. Giaume C, Kirchhoff F, Matute C, Reichenbach A, Verkhratsky A. Glia: the fulcrum of brain diseases. Cell Death Differ. 14(7), 1324-1335 (2007).

42. Tian L, Hui CW, Bisht K et al. Microglia under psychosocial stressors along the aging trajectory: Consequences on neuronal circuits, behavior, and brain diseases. Prog. Neuropsychopharmacol. Biol. Psychiatry

doi:10.1016/j.pnpbp.2017.01.007 (2017).

43. Tay TL, Savage JC, Hui CW, Bisht K, Tremblay ME. Microglia across the lifespan: from origin to function in brain development, plasticity and cognition. J. Physiol. 595(6), 1929-1945 (2017).

44. Kovacs GG, Lee VM, Trojanowski JQ. Protein astrogliopathies in human neurodegenerative diseases and aging. Brain Pathol. 27(5), 675-690 (2017).

45. Verkhratsky A, Marutle A, Rodriguez-Arellano JJ, Nordberg A. Glial asthenia and functional paralysis: A new perspective on neurodegeneration and Alzheimer's disease. Neuroscientist 21(5), 552-568 (2015).

46. Beauquis J, Pavia P, Pomilio C et al. Environmental enrichment prevents astroglial pathological changes in the hippocampus of APP transgenic mice, model of Alzheimer's disease. Exp. Neurol. 239 28-37 (2013).

47. Beauquis J, Vinuesa A, Pomilio C, Pavia P, Galvan V, Saravia F. Neuronal and glial alterations, increased anxiety, and cognitive impairment before hippocampal amyloid deposition in PDAPP mice, model of Alzheimer's disease. Hippocampus 24(3), 257-269 (2014).

48. Kulijewicz-Nawrot M, Verkhratsky A, Chvatal A, Sykova E, Rodriguez JJ. Astrocytic cytoskeletal atrophy in the medial prefrontal cortex of a triple transgenic mouse model of Alzheimer's disease. J. Anat. 221(3), 252-262 (2012).

49. Olabarria M, Noristani HN, Verkhratsky A, Rodriguez JJ. Concomitant astroglial atrophy and astrogliosis in a triple transgenic animal model of Alzheimer's disease. Glia 58 831-838 (2010).

$\bullet \bullet$ first demonstration of early astroglial atrophy in AD rodent model

50. Yeh CY, Vadhwana B, Verkhratsky A, Rodriguez JJ. Early astrocytic atrophy in the entorhinal cortex of a triple transgenic animal model of Alzheimer's disease. ASN Neuro 3(5), 271-279 (2011).

51. Heneka MT, Sastre M, Dumitrescu-Ozimek L et al. Focal glial activation coincides with increased BACE1 activation and precedes amyloid plaque deposition in APP[V717I] transgenic mice. J. Neuroinflammation 222 (2005).

52. Rodriguez-Vieitez E, Saint-Aubert L, Carter SF et al. Diverging longitudinal changes in astrocytosis and amyloid PET in autosomal dominant Alzheimer's disease. Brain 139(Pt 3), 922-936 (2016). 
53. Bales KR. The value and limitations of transgenic mouse models used in drug discovery for Alzheimer's disease: an update. Expert Opin. Drug Discov. 7(4), 281-297 (2012).

• this paper sets the context as to why new ways of modelling AD are essential for therapy development.

54. Onos KD, Sukoff Rizzo SJ, Howell GR, Sasner M. Toward more predictive genetic mouse models of Alzheimer's disease. Brain Res. Bull. 122 1-11 (2016).

55. Gotz J, Ittner LM. Animal models of Alzheimer's disease and frontotemporal dementia. Nat. Rev. Neurosci. 9(7), 532-544 (2008).

56. Zhang Y, Sloan SA, Clarke LE et al. Purification and Characterization of Progenitor and Mature Human Astrocytes Reveals Transcriptional and Functional Differences with Mouse. Neuron 89(1), 37-53 (2016).

57. Yu J, Vodyanik MA, Smuga-Otto K et al. Induced pluripotent stem cell lines derived from human somatic cells. Science 318(5858), 1917-1920 (2007).

58. Takahashi K, Yamanaka S. Induction of pluripotent stem cells from mouse embryonic and adult fibroblast cultures by defined factors. Cell 126(4), 663676 (2006).

- seminal paper in the field - first description of iPSC creation from somatic cells; identified the transcription factors central to restoring potency to differentiated cells (collectively known at the "Yamanaka factors")

59. Takahashi K, Tanabe K, Ohnuki M et al. Induction of pluripotent stem cells from adult human fibroblasts by defined factors. Cell 131(5), 861-872 (2007).

60. Israel MA, Yuan SH, Bardy $\mathrm{C}$ et al. Probing sporadic and familial Alzheimer's disease using induced pluripotent stem cells. Nature 482(7384), 216-220 (2012).

- first paper using patient iPSC-derived cells (neurones) to investigate AD in vitro. Validates the use of iPSC-derived neural cells for this purpose.

61. Shi Y, Kirwan P, Livesey FJ. Directed differentiation of human pluripotent stem cells to cerebral cortex neurons and neural networks. Nat. Protoc. 7(10), 1836-1846 (2012).

62. Mohamet L, Miazga NJ, Ward CM. Familial Alzheimer's disease modelling using induced pluripotent stem cell technology. World J. Stem. Cells 6(2), 239-247 (2014).

- comprehensive review of the use of iPSC-derived neurones for modelling AD in vitro.

63. Shaltouki A, Peng J, Liu Q, Rao MS, Zeng X. Efficient generation of astrocytes from human pluripotent stem cells in defined conditions. Stem Cells 31(5), 941-952 (2013).

64. Krencik R, Zhang SC. Directed differentiation of functional astroglial subtypes from human pluripotent stem cells. Nat. Protoc. 6(11), 1710-1717 (2011).

65. Roybon L, Lamas NJ, Garcia AD et al. Human stem cell-derived spinal cord astrocytes with defined mature or reactive phenotypes. Cell Rep. 4(5), 10351048 (2013).

66. Kondo T, Asai M, Tsukita K et al. Modeling Alzheimer's disease with iPSCs reveals stress phenotypes associated with intracellular Abeta and differential drug responsiveness. Cell Stem Cell 12(4), 487-496 (2013).

- this article documents altered responses to ER and oxidative stress-relieving compounds in embryonic stem cell versus iPSC-derived astrocytes. 
67. Liao MC, Muratore CR, Gierahn TM et al. Single-Cell Detection of Secreted $\mathrm{A} \beta$ and sAPP $\alpha$ from Human IPSC-Derived Neurons and Astrocytes. $J$. Neurosci. 36(5), 1730-1746 (2016).

68. Emdad L, D'souza SL, Kothari HP, Qadeer ZA, Germano IM. Efficient differentiation of human embryonic and induced pluripotent stem cells into functional astrocytes. Stem Cells Dev. 21(3), 404-410 (2012).

69. Hallmann AL, Arauzo-Bravo MJ, Mavrommatis L et al. Astrocyte pathology in a human neural stem cell model of frontotemporal dementia caused by mutant TAU protein. Sci Rep. 742991 (2017).

70. Rao MS, Noble M, Mayer-Proschel M. A tripotential glial precursor cell is present in the developing spinal cord. Proc. Natl. Acad. Sci. U. S. A. 95(7), 3996-4001 (1998).

71. Santos R, Vadodaria KC, Jaeger BN et al. Differentiation of InflammationResponsive Astrocytes from Glial Progenitors Generated from Human Induced Pluripotent Stem Cells. Stem Cell Rep. 8(6), 1757-1769 (2017).

72. Jones VC, Atkinson-Dell R, Verkhratsky A, Mohamet L. Aberrant iPSCderived human astrocytes in Alzheimer's disease. Cell Death Dis. 8(3), e2696 (2017).

- this article describes a robust and efficient accelerated method for the directed differentiation of healthy, FAD- and SAD patient-derived iPSCs to mature astrocytes in vitro. The authors also use this platform to demonstrate human astroglial atrophy in both forms of $\mathrm{AD}$.

73. Bonni A, Sun Y, Nadal-Vicens M et al. Regulation of gliogenesis in the central nervous system by the JAK-STAT signaling pathway. Science 278(5337), 477-483 (1997).

74. Nakashima K, Yanagisawa M, Arakawa $\mathrm{H}$ et al. Synergistic signaling in fetal brain by STAT3-Smad1 complex bridged by p300. Science 284(5413), 479482 (1999).

75. Gross RE, Mehler MF, Mabie PC, Zang Z, Santschi L, Kessler JA. Bone morphogenetic proteins promote astroglial lineage commitment by mammalian subventricular zone progenitor cells. Neuron $17(4), 595-606$ (1996).

76. Michler-Stuke A, Wolff JR, Bottenstein JE. Factors influencing astrocyte growth and development in defined media. Int. J. Dev. Neurosci. 2(6), 575584 (1984).

77. Sanalkumar R, Vidyanand S, Lalitha Indulekha C, James J. Neuronal vs. glial fate of embryonic stem cell-derived neural progenitors (ES-NPs) is determined by FGF2/EGF during proliferation. J. Mol. Neurosci. 42(1), 17-27 (2010).

78. Emsley JG, Macklis JD. Astroglial heterogeneity closely reflects the neuronaldefined anatomy of the adult murine CNS. Neuron Glia Biol. 2(3), 175-186 (2006).

79. Chai H, Diaz-Castro B, Shigetomi E et al. Neural Circuit-Specialized Astrocytes: Transcriptomic, Proteomic, Morphological, and Functional Evidence. Neuron 95(3), 531-549 e539 (2017).

80. Hol EM, Pekny M. Glial fibrillary acidic protein (GFAP) and the astrocyte intermediate filament system in diseases of the central nervous system. Curr. Opin. Cell. Biol. 32 121-130 (2015).

81. Tcw J, Wang M, Pimenova AA et al. An efficient platform for astrocyte differentiation from human induced pluripotent stem cells. Stem Cell Rep. 9(2), 600-614 (2017). 
82. Liu H, Zhang SC. Specification of neuronal and glial subtypes from human pluripotent stem cells. Cell. Mol. Life. Sci. 68(24), 3995-4008 (2011).

83. Verkhratsky A, Zorec R, Parpura V. Stratification of astrocytes in healthy and diseased brain. Brain Pathol. 27(5), 629-644 (2017).

84. Oksanen M, Petersen AJ, Naumenko N et al. PSEN1 Mutant iPSC-Derived Model Reveals Severe Astrocyte Pathology in Alzheimer's Disease. Stem Cell Rep. 9(6), 1885-1897 (2017).

- paper demonstarting that iPSC-derived $\mathrm{AD}$ astrocytes have aberrant $\mathrm{Ca}^{2+}$ signalling, altered cytokine secretion and increased production of reactive oxygen species.

85. Zhao J, Davis MD, Martens YA et al. APOE epsilon4/epsilon4 diminishes neurotrophic function of human iPSC-derived astrocytes. Hum. Mol. Genet. 26(14), 2690-2700 (2017).

$\bullet \bullet$ paper demonstrating that astrocytes derived from $\mathrm{ApoEA}^{+/+}$individual iPSCs are impaired in their ability astrocytes show impaired abilities to support neuronal survival and synaptogenesis.

86. Scimemi A, Beato M. Determining the neurotransmitter concentration profile at active synapses. Mol. Neurobiol. 40(3), 289-306 (2009).

87. Corder EH, Saunders AM, Strittmatter WJ et al. Gene dose of apolipoprotein E type 4 allele and the risk of Alzheimer's disease in late onset families. Science 261(5123), 921-923 (1993).

88. Corder EH, Saunders AM, Risch NJ et al. Protective effect of apolipoprotein E type 2 allele for late onset Alzheimer disease. Nat. Genet. 7(2), 180-184 (1994).

89. Bu G. Apolipoprotein E and its receptors in Alzheimer's disease: pathways, pathogenesis and therapy. Nat. Rev. Neurosci. 10(5), 333-344 (2009).

90. Hanson AJ, Bayer-Carter JL, Green PS et al. Effect of apolipoprotein E genotype and diet on apolipoprotein E lipidation and amyloid peptides: randomized clinical trial. JAMA Neurol. 70(8), 972-980 (2013).

91. Youmans KL, Tai LM, Nwabuisi-Heath E et al. APOE4-specific changes in Abeta accumulation in a new transgenic mouse model of Alzheimer disease. $J$. Biol. Chem. 287(50), 41774-41786 (2012).

92. Burbaeva G, Boksha IS, Tereshkina EB, Savushkina OK, Starodubtseva LI, Turishcheva MS. Glutamate metabolizing enzymes in prefrontal cortex of Alzheimer's disease patients. Neurochem. Res. 30(11), 1443-1451 (2005).

93. Miguel-Hidalgo JJ, Wei J, Andrew M et al. Glia pathology in the prefrontal cortex in alcohol dependence with and without depressive symptoms. Biol. Psychiatry 52(12), 1121-1133 (2002).

94. Revett TJ, Baker GB, Jhamandas J, Kar S. Glutamate system, amyloid ss peptides and tau protein: functional interrelationships and relevance to Alzheimer disease pathology. J. Psychiatry Neurosci. 38(1), 6-23 (2013).

95. Kulijewicz-Nawrot M, Sykova E, Chvatal A, Verkhratsky A, Rodriguez JJ. Astrocytes and glutamate homoeostasis in Alzheimer's disease: a decrease in glutamine synthetase, but not in glutamate transporter-1, in the prefrontal cortex. ASN Neuro 5(4), 273-282 (2013).

96. Thorne N, Malik N, Shah S et al. High-Throughput Phenotypic Screening of Human Astrocytes to Identify Compounds That Protect Against Oxidative Stress. Stem Cells Transl. Med. 5(5), 613-627 (2016).

97. Meyer K, Ferraiuolo L, Miranda CJ et al. Direct conversion of patient fibroblasts demonstrates non-cell autonomous toxicity of astrocytes to motor 
neurons in familial and sporadic ALS. Proc. Natl. Acad. Sci. U. S. A. 111(2), 829-832 (2014).

98. Pei Y, Peng J, Behl M et al. Comparative neurotoxicity screening in human iPSC-derived neural stem cells, neurons and astrocytes. Brain Res. $1638(\mathrm{Pt}$ A), 57-73 (2016). 


\section{Tables}

Table 1. Pathological signatures of iPSC-derived astrocytes obtained from donors with AD.

\begin{tabular}{|c|c|c|}
\hline $\mathrm{AD}$ form & Pathological signature & Reference \\
\hline $\begin{array}{l}\text { FAD } \\
\text { PSEN1 } 1 \text { E9 mutation }\end{array}$ & $\begin{array}{l}\text { iPSC-derived AD astrocytes } \\
\text { have aberrant } \mathrm{Ca}^{2+} \text { signalling, } \\
\text { altered cytokine secretion, } \\
\text { increased production of reactive } \\
\text { oxygen species and decreased } \\
\text { production of lactate. }\end{array}$ & {$[84]$} \\
\hline $\begin{array}{l}\text { Clinically healthy } \\
\text { individuals carrying } \\
\text { APOE4 alleles }\end{array}$ & $\begin{array}{l}\text { APOE4 iPSC-derived astrocytes } \\
\text { have reduced supportive and } \\
\text { neurotrophic functions. }\end{array}$ & {$[85]$} \\
\hline $\begin{array}{l}\text { FAD } \\
\text { APP-E693 }) \text { ) and APP- } \\
\text { V717L) mutations } \\
\text { SAD }\end{array}$ & $\begin{array}{l}\text { iPSC-derived AD astrocytes } \\
\text { showed } \beta \text {-amyloid intracellular } \\
\text { accumulations. AD astrocytes } \\
\text { also demonstrated signs of ER } \\
\text { stress and increased levels of } \\
\text { reactive oxygen species. }\end{array}$ & {$[66]$} \\
\hline $\begin{array}{l}\text { FAD (PSEN1 M146L } \\
\text { mutation) } \\
\text { SAD (homozygous } \\
\text { APOE4 alleles) }\end{array}$ & $\begin{array}{l}\text { iPSC-derived AD astrocytes } \\
\text { have atrophic morphology as } \\
\text { well as aberrant expression and } \\
\text { localisation of glutamate } \\
\text { transporter EAAT1, glutamate } \\
\text { synthetase and S100B protein. } \\
\text { AD astrocytes also had } \\
\text { decreased constitutive secretion } \\
\text { of soluble inflammatory } \\
\text { mediators. }\end{array}$ & {$[72]$} \\
\hline
\end{tabular}




\section{Figure legends}

Figure 1. Principles of stem cell technology

Human induced pluripotent stem cells (iPSCs), non-invasively generated from somatic cells (e.g. skin fibroblasts), have the capacity to self-renew indefinitely, can generate all cells of the body and retain the genetic information of the donor. Robust generation of specific mature neural cells (such as astroglia) from iPSCs created from patients with brain pathologies offer an unrivalled platform for the study of human brain disorders in vitro, including the screening of potential novel therapeutics, more accurate disease modelling and in cellular and personalised therapy.

Figure 2. Schematic diagram showing astrocyte differentiation from human iPSCs.

A: The differentiation of astrocytes from iPSC colonies follows the early neuronal developmental progress through adherent culture stages over 30 days (from left to right).

B: An undifferenatiated iPSC colony immunostained for markers of pluripotency (Oct3/4 is shown in red and the cell surface marker TRA-180 in green). Initial directed differentiation of pluripotent iPSCs towards neural induction is triggered by serum induction and SMAD inhibition for 6-7 days.

C: iPSC-derived neural stem cells (NSCs) form polarised rosettes expressing canonical markers such as nestin (shown here in green). Following NSC expansion for a further 6-7 days, glial progenitor formation is induced by the supplementation of EGF, BMP-2, CTNF and insulin.

D: By day 14, glial commitment is achieved, and after 30 days astrocytes express classical markers of nature astroglial cells GFAP, protein S100B, glutamate transporter EAAT1 and glutamine synthetase GS. Immunostaining is shown in green, DAPI counterstained nuclei are shown in blue. Scale bars $=50 \mu \mathrm{m}$ (Inset; $20 \mu \mathrm{m}$ ).

Panel D is Reproduced from [72].

Figure 3. Comparison of healthy, FAD and SAD patient-derived $\beta$-III-tubulin immunoreactive neurones and GFAP immunoreactive astrocytes.

Under terminal neuronal differentiation conditions for 35-40 days, all patient samples showed positive expression of the neural marker $\beta$-III-tubulin (green) no significant difference in the proportion of $\beta$-III-tubulin+ neurones between any individual was observed $\left(\mathrm{N}=4\right.$ per group, ANOVA, $\left.\mathrm{F}_{(2,9)}=0.128, \mathrm{p}=\mathrm{ns}\right)$.

In contrast, AD-astrocytes derived from identical patient NPCs show markedly reduced heterogeneity of morphology and striking atrophy compared to healthy cells (GFAP; green). These findings validate that atrophic astroglia do not arise as a result of defective neuronal intermediates in either form of $\mathrm{AD}$, but as a cell-autonomous event. Scale bar $=50 \mu \mathrm{m}$,

Reproduced from [72] 